\title{
EchoGéo
}

$10 \mid 2009$

La piraterie

\section{Le rôle des sociétés de sécurité privées dans la sécurisation du détroit de Malacca (2000-2008)}

Témoignage

Gilles Bonavita

\section{OpenEdition}

Journals

Édition électronique

URL : https://journals.openedition.org/echogeo/11438

DOI : 10.4000/echogeo. 11438

ISSN : 1963-1197

Éditeur

Pôle de recherche pour l'organisation et la diffusion de l'information géographique (CNRS UMR 8586)

Référence électronique

Gilles Bonavita, «Le rôle des sociétés de sécurité privées dans la sécurisation du détroit de Malacca (2000-2008) », EchoGéo [En ligne], 10 | 2009, mis en ligne le 14 octobre 2009, consulté le 16 septembre 2021. URL : http://journals.openedition.org/echogeo/11438; DOI : https://doi.org/ 10.4000/echogeo. 11438

Ce document a été généré automatiquement le 16 septembre 2021

EchoGéo est mis à disposition selon les termes de la licence Creative Commons Attribution - Pas d'Utilisation Commerciale - Pas de Modification 4.0 International (CC BY-NC-ND) 


\title{
Le rôle des sociétés de sécurité privées dans la sécurisation du détroit de Malacca (2000-2008)
}

Témoignage

\author{
Gilles Bonavita
}

1 Le détroit de Malacca et la mer de Chine ont longtemps été des terrains d'insécurité reconnus du monde entier parce qu'infestés de pirates. Aujourd'hui, le détroit de Malacca est un des passages stratégiques du commerce international avec plus de 50000 navires y transitant annuellement ${ }^{1}$. C'est la route la plus courte ente l'Asie et l'Europe et c'est aussi l'axe d'approvisionnement majeur des pays de l'Asie de l'Est pour les matières énergétiques. Zone historiquement la plus dangereuse du monde en matière de sûreté, elle est à présent éclipsée par la piraterie qui sévit dans le golfe d'Aden et au large de la Corne de l'Afrique. Depuis le mois d'octobre 2006, le détroit de Malacca n'est plus qualifié de « route maritime dangereuse » par la Loyd's Registrer (site de référence en matière de statistiques maritimes²). Les statistiques du Regional Cooperation Agreement on Combating Piracy and Armed Robbery Against Ships in Asia's (ReCAAP) ne mentionnent qu'une seule attaque dans le détroit de Malacca pour le premier semestre $2009^{3}$.

2 En juillet 2005, une coopération des Etats riverains a vu naitre le RMSI : Regional Maritime Security Initiative. La Malaisie, Singapour et l'Indonésie coopèrent d'ores et déjà dans le domaine de la surveillance et de la sécurisation des détroits, notamment par l'installation de radars et la mise en place de patrouilles aéromaritimes. La Thaïlande vient de s'associer à ce dispositif de surveillance. Si la Malaisie rejette fermement les initiatives de certains armateurs visant à faire escorter leurs bâtiments par des hommes en armes, les pays riverains s'opposent globalement à toute participation étrangère aux patrouilles. En 2003, la proposition des Etats-Unis de participer à la surveillance de la zone avait reçu une fin de non-recevoir de la part des pays concernés. Pour autant, ceux-ci sont intéressés par une assistance technique qui se manifeste, 
aujourd'hui, par la participation australienne à deux centres, situés en Indonésie et en Malaisie, et destinés davantage à la lutte contre le terrorisme que contre la piraterie.

Photo 1 - Patrouilleur offert par le Japon à l'Indonésie

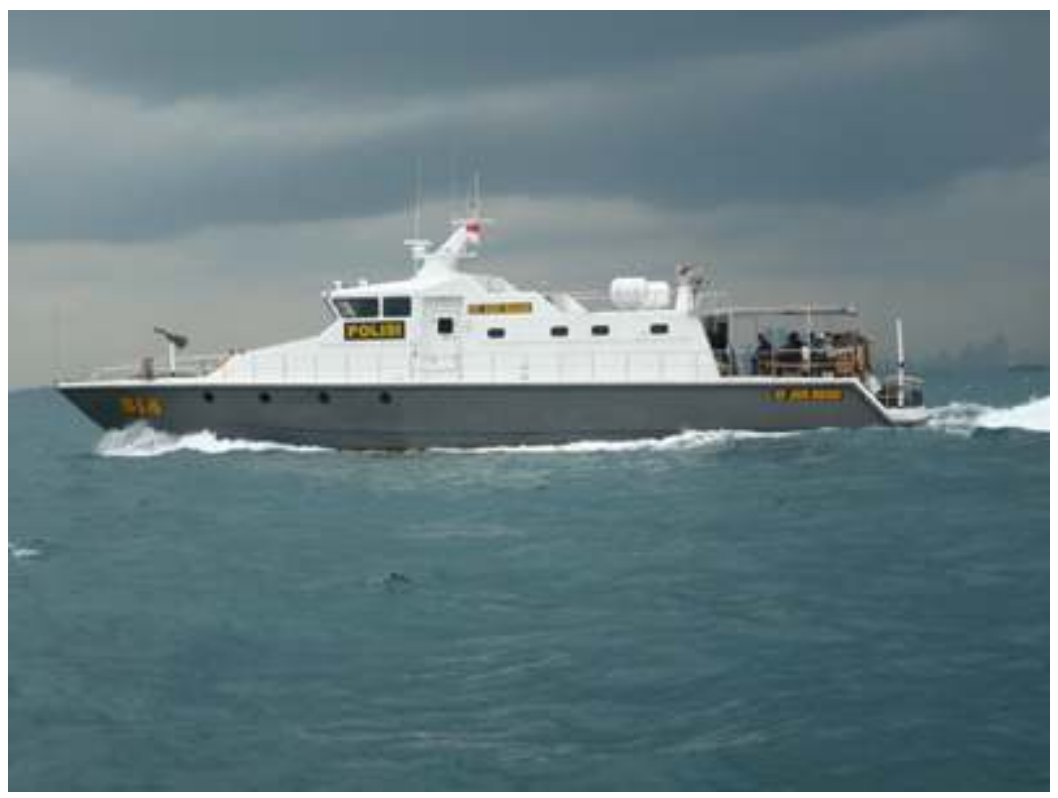

Cliché d'Eric Frécon

3 A ces dispositifs étatiques parfaitement médiatisés, il convient d'ajouter l'amélioration de la bonne gouvernance de certains pays et l'effet post-tsunami qui a vu, outre la perte d'outils de travail des pirates, les puissances internationales venir en a ide aux populations en Aceh. Cette province indonésienne, où la piraterie était source de revenus pour la vie quotidienne et toutes sortes de trafics, a changé complètement de physionomie depuis les accords de 2005 entre le gouvernement indonésien et les mouvements séparatistes.

4 Bien que le sujet soit très sensible au regard de la souveraineté des Etats, il semble que le recours à des sociétés de sécurité, souvent sponsorisées par des puissances étrangères, notamment les Etats-Unis d'Amérique, ayant d'importants intérêts dans la zone, ait favorisé et accéléré la sécurisation du transit des navires dans les détroits. Il paraît probable que ces compagnies de sécurité privées ont participé à l'amélioration de la sécurité dans les détroits malgré de nombreuses critiques qui y voyaient l'acte de mercenaires.

\section{Les craintes des armateurs dans le détroit de Malacca}

$5 \quad$ Les différents types d'incidents, dus à des actes de piraterie, observés par les navires qui ont transité dans les détroits durant la période 2000-2005 peuvent être classés en quatre catégories :

- l'incident mineur, très aléatoire qui peut dégénérer et devenir violent ;

- le vol en mer ;

- la prise ou l'enlèvement avec demande rançon ;

- l'acte terroriste. 
6 Le premier type d'incident est imputable à l'exiguïté de la zone. La présence de nombreux pêcheurs et le transit de grands navires ou encore de grands dispositifs attelés endommagent les matériels de ces pêcheurs qui, excédés, peuvent avoir des réactions violentes et armées.

7 La deuxième catégorie est la plus commune. Elle vise à intercepter des navires lents, peu éloignés des côtes et facilement abordables pour monter à bord et voler les équipages. Les butins obtenus ne sont jamais très importants mais le risque demeure avec l'utilisation d'armes à feux voire la prise d'otage. La frontière avec le sentiment d'un acte terroriste est alors bien mince.

8 La crainte majeure des capitaines de navire vis-à-vis de la piraterie reste le kidnapping. Le fait que des rançons soient généralement payées tend à prouver que cette action est rémunératrice pour leurs auteurs.

9 L'acte terroriste dont la menace est régulièrement évoquée par les autorités est un élément majeur dans les mises en place d'actions de coordination et de surveillance des pays riverains. Cependant aucun incident de terrorisme maritime dans les détroits n'a été observé à ce jour. Certes, des groupes terroristes peuvent utiliser la piraterie comme source de revenus pour leurs activités mais il n'y a pas d'action politique qui ait motivé une action violente contre des objectifs maritimes. Les objectifs potentiels sont nombreux dont certains sont de grandes dimensions et se déplacent lentement : une fois aux mains de terroristes la reprise du contrôle serait particulièrement difficile. On comprend la volonté des Etats d'éradiquer la piraterie qui pourrait être un véritable terreau pour des activités terroristes. Aussi longtemps que les agences gouvernementales et forces de souveraineté exerceront un contrôle sur les mouvements des individus, les mouvements de fonds, les sources d'approvisionnement en munitions et explosifs ainsi que sur les moyens de communication individuels, il sera très difficile pour des terroristes de mener une attaque sur un navire transitant dans le détroit de Malacca.

10 La plupart des compagnies de navigation qui utilisent le concours de sociétés privées le font en raison du risque terroriste et des conséquences qu'aurait une attaque pour leurs personnels et leur survie économique. Bien qu'elles reconnaissent que le risque d'une telle attaque soit faible, les conséquences seraient d'une telle ampleur que le surcoût de la dépense en s'octroyant le concours de sociétés privées est considéré comme marginal.

\section{Quels types de recours aux sociétés privées de sécurité en mer?}

11 Ces sociétés privées effectuent une sécurité maritime tactique avec des services souvent onéreux car il est nécessaire d'engager de lourds moyens matériels (affrètement de navires, d'hélicoptères...) et humains (équipages, recrutement d'anciens militaires occidentaux et asiatiques, développement du renseignement, recrutement et formation de Gurkhas...) pour garantir le succès de l'opération.

12 Les compagnies clientes présentent une certaine similarité :

- les navires ou attelages remorqués qui sont lents et vulnérables ;

- les navires qui ont une grande valeur marchande ou un chargement à protéger ;

- les personnels embarqués qui peuvent être nombreux et très spécialisés ; 
- les navires qui sont sous la pression de lourdes pénalités en cas de non respect des délais.

13

En fait, ces compagnies mettant en œuvre ces navires de transport ont, de la même manière que pour des opérations à terre, besoin de concours extérieurs complémentaires et spécialisés afin de prendre toutes les précautions nécessaires pour un transit de biens à haute valeur ajoutée (transport de munitions, d'infrastructures industrielles, etc.).

14 Les sociétés de sécurités privées en mer ne viennent aucunement se substituer aux organisations gouvernementales en place mais en supplément des moyens fournis par les marines, garde-côtes ou polices.

15 Ces sociétés privées paraissent avoir un effet dissuasif et fournir une première réponse de la même manière que le font les gardes armées devant les banques. La grande différence demeure dans le temps de réponse qui sur mer est plus long en particulier pour le ralliement de moyens étatiques. Pour cette raison il semble que l'objectif des sociétés privées, qui ont opéré dans les détroits au début de la décennie, a été de dissuader toute attaque aussi longtemps que possible ou d'obliger d'éventuels attaquants à battre en retraite avant l'intervention de forces de souveraineté. Ces sociétés de sécurité privées ne cherchent pas à se positionner au-dessus des lois ni même à éradiquer le fléau de la piraterie. Leur rôle a été semble-t-il de se positionner en supplément des forces existantes et de jouer un rôle de dissuasion tout en rassurant les équipages.

16 Dans le cas du détroit de Malacca, Singapour, la Malaisie et l'Indonésie ont chacun effectué des missions de surveillance dans des zones de responsabilité nationale ainsi que des patrouilles aéromaritimes en équipages mixtes afin de partager au mieux l'information. Elles n'ont pour autant pas assuré des fonctions d'escorte comme le faisaient les sociétés privées.

\section{Un calme revenu dans le détroit de Malacca}

17 Le détroit de Malacca, point de passage majeur pour le commerce mondial dans une région économique en pleine expansion c'est-à-dire bien adaptée aux prédateurs, est devenu une zone paisible. Au contraire, dans le golfe d'Aden, les marines régulières des plus grands pays font face à des pirates somaliens parfaitement organisés.

La géographie explique cette amélioration de la situation dans un détroit qui est toujours plus facile à surveiller et contrôler qu'un golfe. La raison est aussi économique. Les pays riverains du détroit de Malacca dépendent totalement de ce passage. Ils ont eu la volonté politique de se donner les moyens pour le sécuriser notamment en lançant des campagnes de communication, en médiatisant à outrance leurs activités de surveillance et en impliquant beaucoup de pays intéressés pour des aides et des actions non militaires. C'est cette action non militaire qui semble avoir réussi. 
Photo 2 - Patrouille indonésienne dans le détroit de Malacca

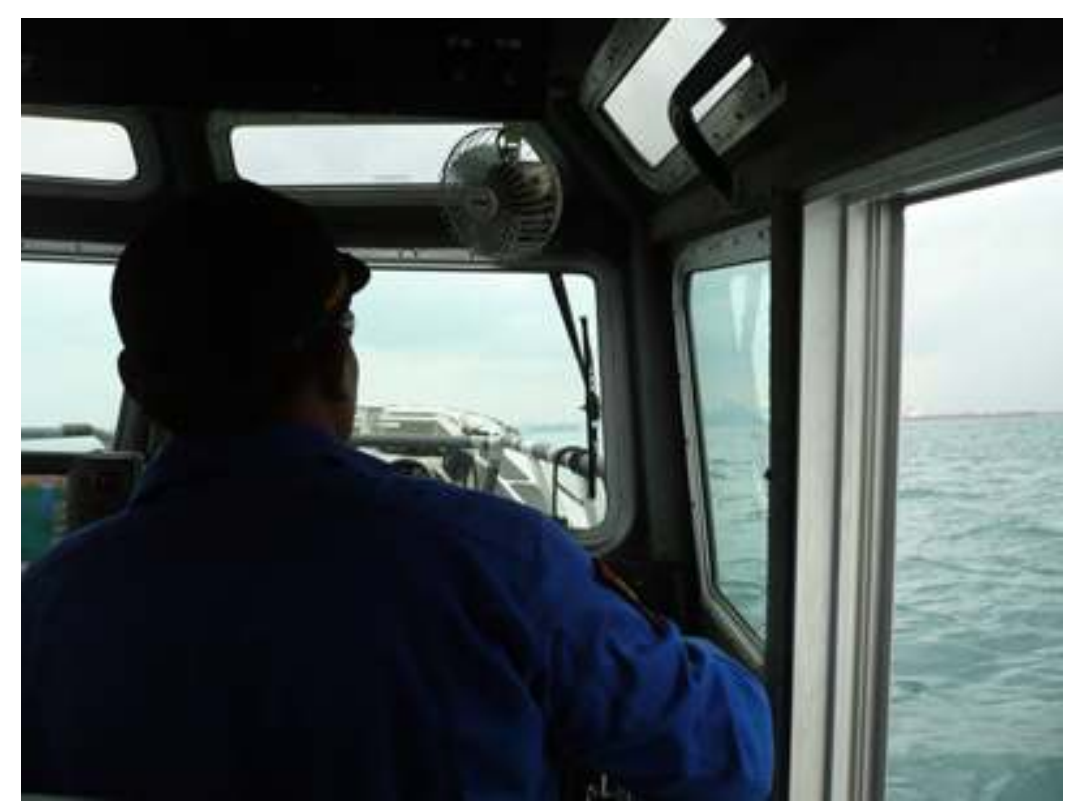

Cliché d'Eric Frécon

19 Les acteurs économiques ont fait parfois appel à des sociétés de sécurité présumées être gérées par de nouveaux mercenaires. Ces sociétés, qui aujourd'hui semblent avoir abandonné une bonne partie de leurs activités de sécurité dans le détroit de Malacca faute de marché, ont longtemps souffert d'une mauvaise réputation, probablement justifiée par leur publicité commerciale outrancière de leur capacité potentielle pour attirer le client vers un marché très lucratif. Néanmoins discrètement soutenues par les pays riverains qui les toléraient et largement rémunérées par des puissances ayant trop d'intérêts dans la zone, elles ont certainement participé au retour de la sérénité dans le détroit de Malacca.

20 La compagnie malaisienne Glenn Defense Marine, qui assure dans le sud-est asiatique un important soutien logistique auprès de nombreuses marines, dont l'US Navy,ainsi que la compagnie Background Asia Risk Solutions (BARS) basée à Singapour, ont été les acteurs privés qui ont œuvré pour la sécurisation du détroit de Malacca. Si Glenn Defense Marine ne semble plus être un acteur majeur dans ce domaine en raison d'une faible activité de piraterie dans le détroit, la société BARS, entièrement spécialisée dans la sécurité maritime en Asie et au Moyen Orient, contribue actuellement, depuis le Yémen, à la sécurisation de la Corne de l'Afrique.

\section{Une sécurisation du détroit de Malacca partiellement privée?}

21 La réussite indéniable de la sécurisation du détroit de Malacca est à mettre au crédit de la bonne gouvernance et de la bonne entente des pays riverains aidés discrètement et financièrement par les utilisateurs aux forts intérêts. Cette réussite tend aussi à démontrer qu'il existe bien des circonstances où les sociétés privées de sécurisation sont envisageables. Un nécessaire contrôle des Etats pour s'assurer du respect des lois en vigueur est à mettre en place. Mais c'est là une réponse non militaire qui est venue 
discrètement s'ajouter à la panoplie de moyens mis en place pour la sécurisation actuelle du détroit de Malacca. On comprend mieux pourquoi, ces derniers mois, les amiraux américains en fonction en mer d'Arabie ont souhaité que des compagnies privées s'impliquent davantage dans la lutte contre la piraterie dans le golfe d'Aden! Malgré des résultats indéniables dans le détroit de Malacca (38 attaques en 2004, 11 en 2006, 2 en 2008 d'après le Bureau maritime international ${ }^{4}$ ), le recours à des sociétés privées reste impopulaire, beaucoup y voyant une forme de mercenariat avec tous ses défauts. La France vient d'apporter une réponse différente en plaçant, aux frais des armateurs, des commandos français à bord des thoniers en campagne de pêche dans l'océan Indien ${ }^{5}$. Beaucoup s'interrogent sur cette pratique. Le débat reste bien ouvert sur les questions de sécurisation maritime face à une piraterie récurrente.

Photo 3 - Type de sampans utilisés par les pirates des Riau - reconstitution (Indonésie)

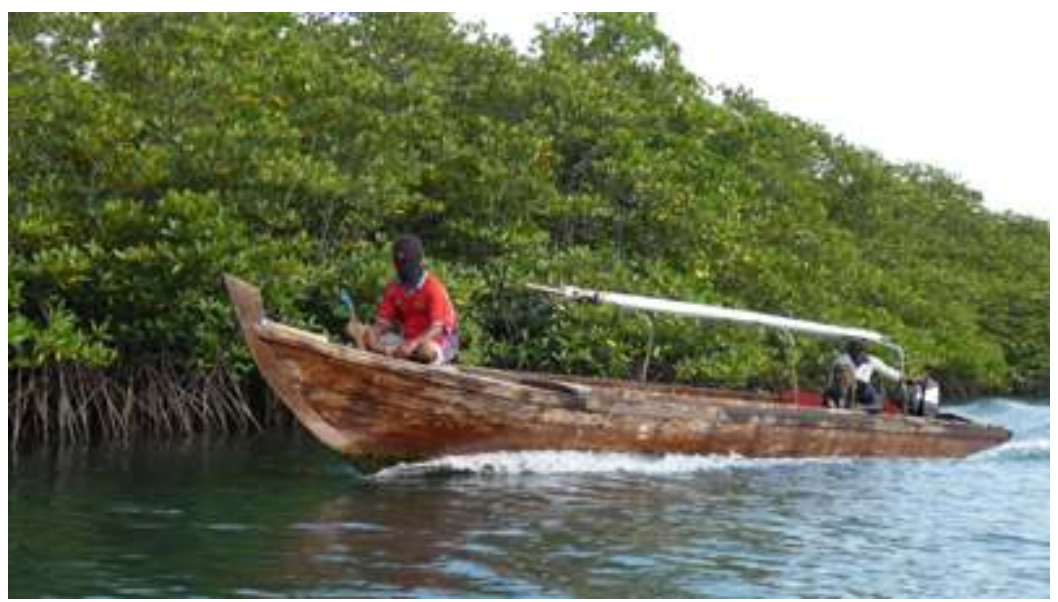

Cliché d'Eric Frécon

\section{NOTES}

1. NTS Centre, August 2009. Maritime Predations in the Malacca Straits: Treading New Waters. Singapore: RSIS, p. 11

2. Voir <http://www.lr.org/> (consulté le 3/8/2009)

3. ReCAAP, July 2009. Half Yearly Report 2009. Singapore: ISC-ReCAAP. 68 p. Voir < http:// www.recaap.org/incident/pdf/reports/2009/ReCAAP\%20ISC\%20Half\%20Yearly\%20Report.pdf> (consulté le 3/8/2009)

4. ICC-IMB, January 2009. Piracy and Armed Robbery against Ships - Annual report - 1/1/2008 31/12/2008. London, ICC-CCS, $47 \mathrm{p}$.

5. Merchet, Jean-Dominique, 6/7/2009. Des fusiliers marins embarquent à bord de thoniers dans l'Océan indien <http://secretdefense.blogs.liberation.fr/defense/2009/07/des-fusiliers-marinsembarquent-\%C3\%A0-bord-de-thoniers-dans-loc\%C3\%A9an-indien/comments/page/2/> (consulté en août 2009) 


\section{RÉSUMÉS}

Le détroit de Malacca, mondialement renommé comme zone active de piraterie, n'est plus considéré comme une zone à risques depuis 2005. S'il est certain que la forte volonté politique des Etats riverains est venue appuyer une mise en œuvre concertée des moyens nationaux avec le soutien des principaux pays utilisateurs, on ne peut écarter le travail discret et certainement efficace mené par des sociétés privées. Impopulaires car perçues comme une forme de mercenariat et critiquées sans être interdites, ces sociétés ne font qu'apporter une réponse à une forte demande des armateurs.

The Strait of Malacca well known all over the world as active area of pirates, found since 2005 a peace in this domain. If it is certain that the strong political willings of the waterside States came to buid a stake in joint work of the national means with the support of major powerful countries, we cannot push aside the discreet and certainly effective work led by private armed maritime security companies; Unpopular, very criticized these companies are only bringing an answer to a strong demand.

\section{INDEX}

Keywords : Armed maritime security services, Malacca Straits, Piracy, Shipping, Somalia Mots-clés : Détroit de Malacca, marine marchande, piraterie, société de sécurité maritime, Somalie

Thèmes : Sur le Champ - Sur le Terrain

\section{AUTEUR}

\section{GILLES BONAVITA}

Gilles Bonavita (gilles.bonavita@gmail.com) est capitaine de vaisseau de réserve et fut attaché de défense français à Singapour de 2005 à 2008. 